\title{
THE LOW-MASS STELLAR LUMINOSITY FUNCTION OF THE 30 DOR STARBURST CLUSTER
}

\author{
HANS ZINNECKER \\ Astrophysikalisches Institut Potsdam \\ An der Sternwarte 16, D-14482 Potsdam, Germany
}

\begin{abstract}
.
Diffraction limited near-infrared H-band $(1.6 \mu \mathrm{m})$ NICMOS HST images are scheduled to be obtained in mid-October 1997 of the young cluster NGC 2070 (age 3.5 Myr) in the 30 Dor giant HII region in the LMC. The aim is to search for the low-mass $\left(M<2 M_{\odot}\right)$ low-luminosity, red preMain Sequence stellar population and to establish the $\mathrm{H}$-band infrared luminosity function. With the NICMOS we can now determine whether the IMF in this prototypical extragalactic starburst cluster is deficient in subsolar low-mass stars or not. The best ground-based data can sample only $\mathrm{M}>2 \mathrm{M}_{\odot}$. In principle, NICMOS in the $\mathrm{H}$-band (F160W) is sensitive enough to reach a magnitude of $\sim 23.5$ in a relatively short integration time, which indeed corresponds to the fantastic possibility to detect young stellar objects with masses near the hydrogen burning limit $\left(\mathrm{M}=0.1 \mathrm{M}_{\odot}\right)$ according to pre-Main Sequence evolutionary models. Even if we could reach only $\mathrm{H}=22.5$ (i.e. $\mathrm{M}=0.4 \mathrm{M}_{\odot}$ ), our observations will still go a long way in directly answering, by star counts, whether the IMF in starburst galaxies is low-mass deficient or not, with all the corresponding far-reaching implications.

The observations would also tell us whether the 30 Dor cluster can be regarded as a prototype young globular cluster. This possibility would be ruled out, if we found NGC 2070 to be low-mass deficient, because old globular clusters do have a rich population of low-mass stars.
\end{abstract}

